\title{
Causes of nursing home placement for older people with dementia: a systematic review and meta-analysis
}

\author{
Sandeep Toot, ${ }^{1}$ Tom Swinson, ${ }^{1}$ Mike Devine, ${ }^{2}$ David Challis ${ }^{3}$ and Martin Orrell ${ }^{4}$ \\ ${ }^{1}$ Research \& Development Department, North East London NHS Foundation Trust, Goodmayes Hospital, Barley Lane, Goodmayes, Essex IG3 8X7, UK \\ ${ }^{2}$ Older Adult Mental Health Team $\mathcal{E}$ Memory Service, North East London NHS Foundation Trust, Broad Street Centre, Morland Road, Dagenham, Essex \\ RM10 9HU, UK \\ ${ }^{3}$ PSSRU, Crawford House (2nd Floor), Precinct Centre, University of Manchester, Booth Street East, Manchester M1 $39 Q S$, UK \\ ${ }^{4}$ Institute of Mental Health, University of Nottingham, Innovation Park, Triumph Road, Nottingham, NG 72 TU, UK
}

Background: Up to half of people with dementia in high income countries live in nursing homes and more than two-thirds of care home residents have dementia. Fewer than half of these residents report good quality of life and most older people are anxious about the prospect of moving into a nursing home. Robust evidence is needed as to the causes of admission to nursing homes, particularly where these risk factors are modifiable.

Methods: We conducted a systematic literature search to identify controlled comparison studies in which the primary outcome was admission to nursing home of older adults with dementia. Identified studies were assessed for validity and 26 (17 cohort and 9 case-control) were included. Qualitative and quantitative analyses were conducted, including meta-analysis of 15 studies.

Results: Poorer cognition and behavioral and psychological symptoms of dementia (BPSD) were consistently associated with an increased risk of nursing home admission and most of our meta-analyses demonstrated impairments in activities of daily living as a significant risk. The effects of community support services were unclear, with both high and low levels of service use leading to nursing home placement. There was an association between caregiver burden and risk of institutionalization, but findings with regard to caregiver depression varied, as did physical health associations, with some studies showing an increased risk of nursing home placement following hip fracture, reduced mobility, and multiple comorbidities.

Conclusion: We recommend focusing on cognitive enhancement strategies, assessment and management of BPSD, and carer education and support to delay nursing home placement.

Key words: older adults, dementia, nursing home placement, risk factors

\section{Introduction}

In 2012, the World Health Organisation and Alzheimer's Disease International emphasized the public health priority status of dementia (WHO, 2012). Recent estimates indicate that 44.4 million people worldwide live with dementia, and this is set to rise to 135.5 million by the year 2050 (Guerchet et al., 2013).

For high income countries, the transition of people with dementia into care homes is a relatively common process; between $33 \%$ and $50 \%$ of people with dementia in high income countries were

Correspondence should be addressed to: Sandeep Toot, Research \& Development Department, North East London NHS Foundation Trust, Goodmayes Hospital, Barley Lane, Goodmayes, Essex IG3 8XF, UK. Phone: +44344 1200555 ext. 64453. Email: Sandeep.toot@nelft.nhs.uk. Received 19 May 2016; revision requested 18 Aug 2016; revised version received 6 Sep 2016; accepted 9 Sep 2016. First published online 3 November 2016. estimated to reside in a care home (Prince et al., 2015). Care home populations are made up of substantial numbers of people with dementia, for example in the UK, it has been estimated that up to $80 \%$ of care home residents have dementia (Alzheimer's Society, 2013). Unfortunately, fewer than half of these residents reported having a good quality of life. Abuse amongst older people in care homes remains a serious problem and most older people are anxious about being admitted to a nursing home, primarily because of concerns about the standard of care they will receive (Cooper et al., 2008). People with dementia and their families frequently experience great difficulties adjusting to the new caring environment in nursing homes and prefer to remain at home for as long as possible (Sury et al., 2013).

In this context, it is helpful to identify factors that facilitate people with dementia remaining at 
home. This systematic review sought to understand the key factors that influence the decision for people with dementia to move into care homes, with particular emphasis on those factors that might be responsive to intervention.

\section{Methods}

\section{Definition of nursing home}

For the purposes of this study, we defined a nursing home as any residential institution in which permanent residents received round the clock care from the staff of the home, which may or may not have included qualified nursing staff. This reflects the majority of our included studies, which used this term in preference to the more generic "care home," which is employed in the UK.

\section{Types of paper included in the review}

Controlled comparison studies, including randomized controlled trials (RCTs), cohort studies, epidemiological studies, case-control studies, systematic literature reviews, and descriptive studies were eligible for inclusion in this review.

\section{Types of risk factor}

The following categories of risk factor for people with dementia resulting in nursing home placement were considered for inclusion in this review: cognitive; behavioral/psychological; carer related; environmental; functional; and physical health related.

\section{Types of comparison group}

In this review, we identified either case-control studies or cohort studies for inclusion. The majority of the case-control studies presented their results as mean and standard deviations or odds ratios. The majority of cohort studies presented their findings as risk ratios or hazard ratios. As a result, the findings in this paper have been presented separately for cohort studies and case-control studies.

\section{Types of outcome measure}

The primary outcomes in this review included the following:

1. number of patients admitted to nursing homes;

2. total number of admissions to nursing homes;

3. time to nursing home placement.

\section{Types of participant}

The inclusion criteria for the participants in this review were that the samples contained:

1. people aged 60 years or older; and

2. people with a dementia diagnosis of any type; or

3. carers of people with dementia.
The exclusion criterion was that none of the participants had a dementia diagnosis or was a carer for someone with dementia.

\section{Search methods for the identification of studies}

ELECTRONIC SEARCHES

We searched the NHS electronic library records, which contained records from the following major healthcare databases: MEDLINE, EMBASE, PsycINFO, CINAHL, Web of Science, and PubMed, for the period 1999 to February 2015.

The following search terms were used for the database searches: old, elder, aged, memory problems, memory disorders, cognition, cognitive disorders, dementia, Alzheimer's, vascular, frontotemporal, predictors, causes, crisis, indicators, risk profiles, risk factors, model, risk assessment, clinical indicators, prediction, trends, forecasting, probability, prevalence, emergency, hospital, psychiatric, hospitalization, patient admission, patient transfer, patient readmission, institutionalization, admissions, nursing home, care home, long-term care.

\section{SEARCHING OTHER RESOURCES}

We checked the reference lists of key papers and relevant systematic reviews identified by the above methods.

\section{Data collection and analysis}

\section{Selection of STUdies}

Titles and abstracts of citations obtained from the search were examined independently by two researchers and any obviously irrelevant articles were discarded. The full text of those studies deemed relevant was obtained. Where it was not possible to accept or reject based on title and abstract alone, the full text of the citation was obtained for further evaluation.

Assessment of eligibility was undertaken from the full text. Attempts were made to obtain additional information from the study authors where necessary. A third reviewer was consulted in cases of doubt over whether or not inclusion criteria were met. In addition, the third reviewer independently reviewed the selected studies and agreement was reached on which papers should be included.

ASSESSMENT OF VALIDITY

Studies meeting the inclusion criteria were assigned a level of evidence according to the NewcastleOttawa Scale (NOS) for assessing the quality of non-randomized studies (case-control and cohort 


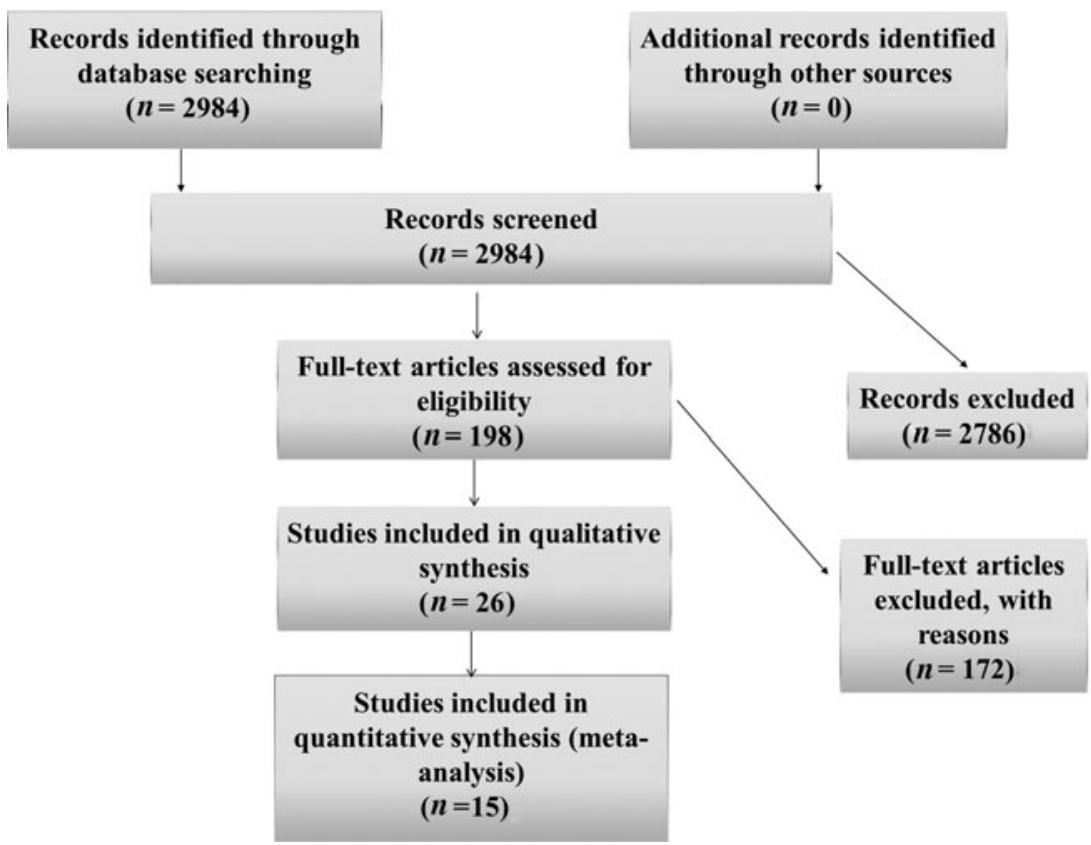

Figure 1. PRISMA flow chart of included and excluded studies.

studies) in meta-analyses (Wells et al., 2012). This consists of a "star system" in which a study is judged on three broad perspectives: the selection of the study groups; the comparability of the groups; and the ascertainment of either the exposure or outcome of interest for case-control or cohort studies respectively. Three reviewers assigned levels of evidence to each study independently and, in cases of disagreement, discussed the studies until a conclusion was agreed. Studies assigned $7^{*}$ or more (maximum 9) were included in this review.

\section{Results}

\section{Included studies}

The PRISMA flowchart (Figure 1) depicts the inclusion and exclusion of studies.

A total of 2,984 references was identified through the literature search, of which 2,786 were excluded by reference to title and abstract alone, as they did not cover risk factors associated with nursing home placement (NHP) for people with dementia.

Of the remaining 198 papers, the full text was obtained and 172 were excluded for the following reasons: sample not appropriate; data not relevant/useful to the review; cross-sectional design only; systematic review; planned/desired NHP rather than actual NHP; end point included placements in a variety of settings, which could not be differentiated; not translated into English; did not assess risk factors for NHP; end point not
NHP; quality rating lower than $7^{*}$ according to the NOS. In light of these exclusions, 26 studies were included in the present review; 17 cohort studies (see Table 1) and 9 case-control studies (see Table 2). Only 15 of these studies could feasibly be included in the meta-analyses.

\section{Cohort studies}

The majority of cohort studies presented their findings as risk ratios (RRs) or hazard ratios (HRs). A RR is a ratio of probabilities of an event between two groups, and represents the relative likelihood of a person experiencing an event if they are part of one group compared with another. A RR should be calculated at the end of a study, using all of the data up until a pre-specified endpoint. A HR represents an instantaneous risk, and hazard ratios may change when calculated at different time points. Studies that report hazard ratios will often use a Cox proportional hazards model, which makes the assumption that a hazard will stay proportionally constant throughout the study. One study (Spruytte et al., 2001) in the present review also reported odds ratios (OR; more commonly used for case-control studies), which are a ratio of the odds of an event between two groups.

The meta-analyses (see Table 3 ) only included those studies that used comparable standardized measures, comparable concepts, and comparable risk indicator data.

When meta-analyzing results from papers that split their risk indicator data into varying degrees of severity, categories indicating moderate severity 
Table 1. Descriptions of cohort studies $(n=17)$

\begin{tabular}{|c|c|c|c|c|c|}
\hline STUDY & COUNTRY & $\begin{array}{l}\text { LEVEL OF EVIDENCE } \\
\text { (NOS STAR RATING) }\end{array}$ & $\begin{array}{l}\text { SAMPLE } \\
\quad \text { SIZE }\end{array}$ & $\begin{array}{c}\text { LENGTH OF } \\
\text { STUDY PERIOD }\end{array}$ & $\begin{array}{c}\text { TYPE OF RISK } \\
\text { INDICATOR DATA }\end{array}$ \\
\hline Andel et al. (2007) & USA & $8^{*}$ & 1,943 & 4 years & Hazard ratios \\
\hline Buhr et al. (2006) & USA & $7^{*}$ & 2,000 & 3 years & Risk ratios \\
\hline Gaugler et al. (2003) & USA & $9^{*}$ & 3,944 & 3 years & Hazard ratios \\
\hline Gaugler et al. (2005) & USA & $9^{*}$ & 3,944 & 3 years & Hazard ratios \\
\hline Gaugler et al. (2000) & USA & $8^{*}$ & 304 & 3 years & Risk ratios \\
\hline Gibbons et al. (2002) & USA & $9^{*}$ & 372 & $\begin{array}{l}12 \text { years } \\
\quad(M=4.4)\end{array}$ & Risk ratios \\
\hline Gilley et al. (2004) & USA & $9^{*}$ & 410 & 4 years & Risk ratios \\
\hline Gilley et al. (2005) & USA & $9^{*}$ & 396 & 3 years & Hazard ratios \\
\hline Harboun et al. (2008) & France & $9^{*}$ & 348 & 5 years & Hazard ratios \\
\hline Kunik et al. (2010) & USA & $7^{*}$ & 215 & 2 years & Hazard ratios \\
\hline Luppa et al. (2012) & Germany & $9^{*}$ & 254 & 6 years & Hazard ratios \\
\hline Scarmeas et al. (2007) & $\begin{array}{l}\text { France/ } \\
\text { Greece/USA }\end{array}$ & $8^{*}$ & 497 & $\begin{array}{l}14 \text { years } \\
\qquad(M=4.4)\end{array}$ & Hazard ratios \\
\hline Severson et al. (1994) & USA & $8^{*}$ & 275 & 4 years & Risk ratios \\
\hline Smith et al. (2000) & USA & $9^{*}$ & 314 & 10 years & Risk ratios \\
\hline Smith et al. (2001) & USA & $8^{*}$ & 985 & $\begin{array}{l}\text { Median } \\
\quad=5.3 \text { years }\end{array}$ & Risk ratios \\
\hline Spruytte et al. (2001) & Belgium & $8^{*}$ & 144 & 6-9 months & Odds ratios \\
\hline Yaffe et al. (2002) & USA & $9^{*}$ & 3,859 & 3 years & Hazard ratios \\
\hline
\end{tabular}

Table 2. Descriptions of case-control studies $(n=9)$

\begin{tabular}{|c|c|c|c|c|c|}
\hline STUDY & COUNTRY & $\begin{array}{l}\text { LEVEL OF EVIDENCE } \\
\text { (NOS STAR RATING) }\end{array}$ & $\begin{array}{l}\text { SAMPLE } \\
\text { SIZE }\end{array}$ & $\begin{array}{l}\text { LENGTH OF } \\
\text { STUDY PERIOD }\end{array}$ & $\begin{array}{c}\text { TYPE OF RISK } \\
\text { INDICATOR DATA }\end{array}$ \\
\hline Coehlo et al. (2007) & USA & $7^{*}$ & 99 & 2 years & Mean + SD \\
\hline De Vugt et al. (2005) & Netherlands & $7^{*}$ & 119 & 2 years & Mean + SD \\
\hline Haupt and Kurz (1993) & Germany & $7^{*}$ & 66 & 1 year & Mean + SD \\
\hline Lieberman and Kramer (1991) & USA & $7^{*}$ & 545 & 1 year & Mean + SD \\
\hline Pot et al. (2001) & Netherlands & $8^{*}$ & 138 & 1 year & Mean \\
\hline Scott et al. (1997) & USA & $7^{*}$ & 786 & 19 months & Odds ratios \\
\hline Steele et al. (1990) & USA & $9^{*}$ & 210 & 3 years & Mean + SD \\
\hline Whitlatch et al. (1999) & USA & $8^{*}$ & 926 & 2 years & Odds ratios \\
\hline Young et al. (1998) & USA & $7^{*}$ & 3,859 & 18 months & Mean \\
\hline
\end{tabular}

were included. If appropriate, risk indicator data were recoded into their reciprocal form $(1 / x)$ in order to ensure the direction of effect was consistent and to include additional studies into the meta-analyses.

\section{Case-control studies}

The majority of the case-control studies presented their results as mean and standard deviations or odds ratios. Only results from standardized measures were included in the meta-analyses (see Table 4).

The outcomes of any meta-analyses using mean and standard deviations are presented as the standard mean difference (SMD): the difference in mean outcome between groups divided by the standard deviation of outcome among participants.
The research team categorized risk factors that were recurrently assessed in the included studies as: cognition; Activities of Daily Living (ADL)/Instrumental Activities of Daily Living (IADL) for the person with dementia; carer burden; Behavioral and Psychological Symptoms of Dementia (BPSD); physical health of person with dementia; carer mental health - depression; other carer factors; and environmental.

\section{Cognition}

COHORT STUDIES

Four studies (Severson et al., 1994; Smith et al., 2001; Gibbons et al., 2002; Gilley et al., 2004) using risk ratios assessed whether poorer cognition influenced the risk of NHP. All four reported that 
Table 3. Cohort studies included in the meta-analyses

\begin{tabular}{|c|c|c|c|c|}
\hline RISK FACTOR & $\begin{array}{l}\text { OUTCOME } \\
\text { MEASURE }\end{array}$ & STUDY & $\begin{array}{l}\text { RISK RATIO }(95 \% \text { CI, } \\
P \text { VALUE })\end{array}$ & $\begin{array}{l}\text { HAZARD RATIO }(95 \% \mathrm{CI} \text {, } \\
P \text { VALUE })\end{array}$ \\
\hline $\begin{array}{l}\text { Poorer } \\
\text { Cognition }\end{array}$ & MMSE & $\begin{array}{l}\text { Gaugler et al. }(2003) \\
\text { Gibbons } \text { et al. }(2002) \\
\text { Gilley et al. }(2004)^{\mathrm{a}} \\
\text { Gilley et al. }(2005)^{\mathrm{a}} \\
\text { Harboun } \text { et al. }(2008) \\
\text { Luppa } \text { et al. }(2012)^{\mathrm{a}} \\
\text { Yaffe } \text { et al. }(2002)\end{array}$ & $\begin{array}{l}- \\
1.45(1.21-1.74, p<0.001) \\
1.05(1.03-1.08, p<0.001) \\
- \\
- \\
- \\
-\end{array}$ & $\begin{array}{l}1.72(1.36-2.17, p<0.001) \\
- \\
- \\
1.03(0.99-1.08, p=0.094) \\
2.23(1.09-4.55, p=0.028) \\
1.09(1.02-1.15, p=0.006) \\
1.52(1.33-1.74, p<0.001)\end{array}$ \\
\hline & DRS & $\begin{array}{l}\text { Severson et al. }(1994) \\
\text { Smith et al. }(2001)^{\mathrm{a}}\end{array}$ & $\begin{array}{l}2.1(1.21-3.58, p=0.007) \\
1.99(1.59-2.49, p<0.001)\end{array}$ & $\begin{array}{l}- \\
-\end{array}$ \\
\hline $\begin{array}{l}\text { Poorer ADL } \\
\text { ability }\end{array}$ & $\begin{array}{l}\text { ADL Checklist } \\
\text { Katz ADL }\end{array}$ & $\begin{array}{l}\text { Gaugler et al. (2000) } \\
\text { Gilley et al. (2004) }\end{array}$ & $\begin{array}{l}1.04(0.93-1.71, p=0.51) \\
0.94(0.82-1.07, p=0.36)\end{array}$ & - \\
\hline $\begin{array}{l}\text { ADL } \\
\text { dependency }\end{array}$ & MDS-HC & $\begin{array}{l}\text { Yaffe et al. (2002) } \\
\text { Harboun et al. (2008) }\end{array}$ & - & $\begin{array}{l}1.3(1.2-1.59, p<0.001) \\
0.9(0.69-1.18, p=0.44)\end{array}$ \\
\hline $\begin{array}{l}\text { ADL/IADL } \\
\text { dependency }\end{array}$ & RIL (Part A) & $\begin{array}{l}\text { Severson et al. (1994) } \\
\text { Smith et al. }(2001)\end{array}$ & $\begin{array}{l}1.7(1.01-2.86, p=0.05) \\
1.74(1.47-2.06, p<0.001)\end{array}$ & - \\
\hline Carer burden & $\begin{array}{l}\text { ZBS } \\
\text { SCB }\end{array}$ & $\begin{array}{l}\text { Gaugler et al. (2003) } \\
\text { Yaffe et al. }(2002) \\
\text { Gilley et al. }(2005)\end{array}$ & $\begin{array}{l}- \\
- \\
-\end{array}$ & $\begin{array}{l}1.28(1.08-1.53, p=0.005) \\
1.21(1.04-1.4, p=0.01) \\
1.05(1.01-1.09, p=0.007)\end{array}$ \\
\hline
\end{tabular}

${ }^{\text {a }}$ Recoded.

MMSE - Mini-Mental State Examination (Folstein et al., 1975); DRS - Dementia Rating Scale (Mattis, 1988); ADL Checklist - Activities of Daily Living Checklist (Lawton and Brody, 1969); Katz ADL - Katz Activities of Daily Living (Katz et al., 1963); MDS-HC -

Minimum Data Set Home Care (Morris et al., 1997); RIL - Record of Independent Living (Weintraub, 1986); ZBS - Zarit Burden Scale (Lawton et al., 1991); SCB - Subjective Caregiving Burden scale (Lawton et al., 1991).

Table 4. Case-control studies included in the meta-analyses

\begin{tabular}{|c|c|c|c|c|c|c|c|}
\hline & & & $\begin{array}{l}\text { MAND SD OF } \\
\text { GROUP } \\
\text { ADMITTED }\end{array}$ & $\begin{array}{l}\text { MAND SD OF } \\
\text { GROUP NOT } \\
\text { ADMITTED }\end{array}$ & STANDARD & & \\
\hline RISK & & OUTCOME & TO NURSING & TO NURSING & DIFFERENCE & STANDARD & \\
\hline FACTOR & STUDY & MEASURE & HOME & HOME & IN MEAN & ERROR & $P$ VALUE \\
\hline \multirow[t]{4}{*}{ Cognition } & $\begin{array}{l}\text { De Vugt et al. } \\
\quad(2005)\end{array}$ & MMSE & $17.6(4.2)$ & $20.1(3.9)$ & -0.061 & 0.22 & 0.005 \\
\hline & $\begin{array}{l}\text { Haupt and Kurz } \\
\text { (1993) }\end{array}$ & CAMCOG & $53.4(21.3)$ & $43.4(13.1)$ & -0.053 & 0.23 & 0.05 \\
\hline & $\begin{array}{l}\text { Lieberman and } \\
\text { Kramer (1991) }\end{array}$ & MMSE & $15.6(6.7)$ & $17.2(8.1)$ & -0.21 & 0.13 & 0.13 \\
\hline & $\begin{array}{l}\text { Steele et al. } \\
\quad(1990)\end{array}$ & MMSE & $9.6(5.8)$ & $12.1(6.7)$ & -0.4 & 0.29 & 0.16 \\
\hline \multirow[t]{4}{*}{ BPSD } & $\begin{array}{l}\text { Coehlo et al. } \\
\text { (2007) }\end{array}$ & NPICG & $22.4(24.2)$ & $10.6(13.2)$ & 0.67 & 0.29 & 0.02 \\
\hline & $\begin{array}{l}\text { De Vugt et al. } \\
\quad(2005)\end{array}$ & NPI & $24.9(22.6)$ & $21.8(22.8)$ & 0.14 & 0.22 & 0.53 \\
\hline & $\begin{array}{l}\text { Lieberman and } \\
\text { Kramer (1991) }\end{array}$ & BRDRS & $5.6(3)$ & $5.2(3.8)$ & 0.11 & 0.13 & 0.41 \\
\hline & $\begin{array}{l}\text { Steele et al. } \\
\quad(1990)\end{array}$ & PGDRS(Beh) & $7.6(5.8)$ & $4.0(4.1)$ & 0.72 & 0.29 & 0.01 \\
\hline \multirow[t]{2}{*}{ ADL } & $\begin{array}{l}\text { Haupt and Kurz } \\
\text { (1993) }\end{array}$ & $\mathrm{BDS}$ & $10.4(5.1)$ & $11.8(3.7)$ & 0.3 & 0.26 & 0.23 \\
\hline & $\begin{array}{l}\text { Steele et al. } \\
\quad(1990)\end{array}$ & PGDRS(ADL) & $11.5(6.4)$ & $6.8(7.5)$ & 0.67 & 0.29 & 0.02 \\
\hline
\end{tabular}

MMSE - Mini-Mental State Examination (Folstein et al., 1975); CAMCOG - Cambridge Cognitive Examination (Roth et al., 1986); NPI - Neuropsychiatric Inventory (Cummings et al., 1994); NPICG - Neuropsychiatric Inventory change score from diagnosis to first follow up; BRDRS - Blessed Roth Dementia Rating Scale (Blessed et al., 1968); PGDRS(Beh) - Psychogeriatric Dependency Rating Scale Behavior subscale (Wilkinson and Graham-White, 1980); BDS - Blessed Dementia Scale (Blessed et al., 1968); PGDRS(ADL) Psychogeriatric Dependency Rating Scale - Activities of Daily Living subscale (Wilkinson and Graham-White, 1980). 
people with dementia with poorer cognition are at greater risk of NHP. A meta-analysis of these studies (see Figure S2) indicated that people with dementia with greater impairment of cognition have a significantly increased risk of NHP (RR = $1.06,95 \%$ CI $1.04-1.09, p<0.001)$.

Five studies using hazard ratios assessed whether poorer cognition influenced the risk of NHP; four (Yaffe et al., 2002; Gaugler et al., 2003; 2005; Harboun et al., 2008; Luppa et al., 2012) reported that people with dementia with poorer cognition are at a greater risk of NHP. However, one study (Gilley et al., 2005) found that poorer cognition did not significantly increase or decrease risk of NHP. In a meta-analysis of these studies (see Figure S3), the results indicated that poorer cognition increases the risk of NHP $(\mathrm{HR}=1.08,95 \%$ CI $1.05-1.18$, $p<0.001)$.

\section{CASE-CONTROL STUDIES}

Five studies assessed whether cognition was a factor in the risk of NHP. Three of these (Haupt and Kurz, 1993; Young et al., 1998; de Vugt et al., 2005) indicated that people with dementia placed in a nursing home have significantly poorer cognition than those who remained in the community. The other two (Steele et al., 1990; Lieberman and Kramer, 1991) reported that there were no significant differences between these two groups.

Four of these studies (Steele et al., 1990; Lieberman and Kramer, 1991; Haupt and Kurz, 1993; de Vugt et al., 2005) were entered into a meta-analysis (see Figure S4) and those people with dementia who were placed in a nursing home had significantly poorer cognition than those who remained in the community $(\mathrm{SMD}=-0.36, \mathrm{SE}=$ $0.1, p<0.001)$.

Young et al. (1998) not included in the metaanalysis, found that people with dementia admitted to a nursing home had poorer verbal ability $(p<0.01)$ and poorer memory $(p<0.001)$ than those not admitted.

\section{ADL or IADL for the person with dementia}

A number of studies looked at ADL or IADL ability as potential risk factors. For clarity, the present review separately analyzed studies looking at ADLs, IADLs, and a combination of ADL/IADLs rather than collating them into a sole ADL/IADL risk factor.

\section{ADL}

\section{COHORT STUDIES}

In the meta-analysis of studies (Gaugler et al., 2000; Gilley et al., 2004) that reported risk ratios (see
Figure S5), poorer ADL ability neither increased nor decreased the risk of NHP (RR $=1.05,95 \%$ CI $0.96-1.15, p=0.27$ ).

In the meta-analysis of studies (Yaffe et al., 2002; Harboun et al., 2008) that reported hazard ratios (see Figure S6), ADL dependency significantly increased the risk of NHP (HR $=1.26,95 \%$ CI $1.11-1.43, p<0.001)$.

Four studies were not included in the metaanalyses and only one of these found that ADL dependency increased the risk of NHP; Smith et al. (2000) found that a change in the level of ADL dependency (from occasional to daily) increased the risk of NHP $(\mathrm{RR}=2.22$, paper states that result is significant but $95 \% \mathrm{CI}$ and $p$ values are not provided).

In contrast, Andel et al. (2007) found that an increase in ADL dependency neither increased nor decreased the risk of NHP (HR $=1, p$ value not provided). Similar findings were reported by Gilley et al. (2005) ( $\mathrm{HR}=0.956,95 \% \mathrm{CI}$ and $p$ value not provided). Buhr et al. (2006) did not find ADL dependencies to be a significant predictor of NHP (no data provided). However, they found that the number of tasks performed for a patient increased the risk of NHP (RR $=1.08,95 \% 1.05-1.11$, $p$ value not provided).

\section{CASE-CONTROL STUDIES}

Five studies investigated whether ADL function for the person with dementia was a risk factor for NHP. Four of these (Steele et al., 1990; Haupt and Kurz, 1993; Scott et al., 1997; Young et al., 1998) reported that ADL were poorer in people with dementia who were placed in a nursing home versus those who remained in the community. One study (de Vugt et al., 2005) found no difference between these two groups.

Two of these studies (Steele et al., 1990; Haupt and Kurz, 1993) were entered into a metaanalysis (see Figure S7) and those people with dementia who were placed in a nursing home had significantly more ADL impairments than those who remained in the community $(\mathrm{SMD}=0.47$, $\mathrm{SE}=0.2, p=0.016)$.

Young et al. (1998) found that people with dementia admitted to a nursing home had significantly greater ADL impairments at the beginning of the study than those who continued to receive care at home $(p<0.01)$. Scott et al. (1997) found that people with dementia who declined in the ability to perform ADL were significantly more likely to be placed in a nursing home compared to those with no decline or an improvement ( $\mathrm{OR}=3.5,95 \%$ CI 1.9-6.4, $p$ value not provided). Conversely, de Vugt et al. (2005) found that ADL 
"initiative" and ADL "performance" did not differ between the two groups ( $p$ values not provided).

\section{IADL}

\section{COHORT STUDIES}

Two studies found that IADL dependencies increased the risk of NHP. Gaugler et al. (2003; 2005) found that only if an individual scored in the higher ranges of IADL dependency ( 6 or more out of 8) was there a significant increase in risk of NHP: $6-6.5$ (HR $=1.69$, no $95 \%$ CI provided, $p=0.01), 7-7.5$ ( $\mathrm{HR}=1.84$, no $95 \%$ CI provided, $p=0.004), 8$ ( $\mathrm{HR}=1.94$, no $95 \%$ CI provided, $p=0.003)$. Harboun et al. (2008) found that IADL dependencies (help with 2 or more) at baseline increased the risk of NHP for people with dementia $(\mathrm{HR}=1.5,95 \%$ CI $1.2-1.9, p=0.001)$.

Two studies found that IADL dependencies neither increased nor decreased the risk of NHP. Buhr et al. (2006) provided no RR, whereas Andel et al. (2007) found $\mathrm{HR}=0.99$ ( $p$ value not provided).

\section{ADL/IADL}

\section{COHORT STUDIES}

Two studies (Severson et al., 1994; Smith et al., 2001) using risk ratios assessed whether combined ADL/IADL dependencies influenced the risk of NHP. Both reported that increased ADL/IADL dependencies increased the risk of NHP. A metaanalysis of these studies (see Figure S8) indicated that increased ADL/IADL dependencies increased the risk of NHP $(\mathrm{RR}=1.7,95 \%$ CI 1.48-2.04, $p<0.001$ ).

\section{Carer burden}

\section{COHORT STUDIES}

Three studies (Yaffe et al., 2002; Gaugler et al., 2003; Gilley et al., 2005) used hazard ratios to assess the influence of carer burden on risk of NHP. All three found that greater caregiver burden increased the risk of NHP. A meta-analysis of these studies (see Figure S9) found a significant increase in risk (HR $=1.07,95 \%$ CI $1.03-1.11, p<0.001)$.

One study (Gaugler et al., 2000) used risk ratios to assess the influence of carer burden on risk of NHP. In this study, "role captivity" significantly increased the risk of NHP ( $\mathrm{RR}=1.14, p=$ $0.01)$. However, "role overload" $(R R=0.94)$ and "worry/strain" $(\mathrm{RR}=1.05)$ did not increase or decrease the risk of NHP (95\% CI and $p$ value not provided).

Another study (Spruytte et al., 2001) used odds ratios to assess the link between carer burden and risk of NHP. This study found that carer burden did not increase or decrease the risk of NHP (data not reported by study author).

\section{CASE-CONTROL STUDIES}

Three studies assessed whether carer burden was a risk factor for NHP. Two of these (Young et al., 1998; Whitlatch et al., 1999) found that carers of people with dementia placed in a nursing home showed significantly higher levels of burden at baseline than carers of people with dementia who remained at home (both studies' results at $p<$ 0.01). Conversely, Haupt and Kurz (1993) found no significant difference between these groups (no $p$ value provided).

\section{BPSD}

COHORT STUDIES

Six studies found that BPSD increased the risk of NHP. Gaugler et al. (2000) found that "problematic behavior" significantly increased the risk of NHP $(\mathrm{RR}=1.10$, no CI data provided, $p<0.01)$. Buhr et al. (2006) found that a unit increase in "behavior dysregulation" ( $\mathrm{RR}=1.07,95 \%$ CI 1.04-1.11, $p$ not provided) and/or "psychotic symptoms" $(\mathrm{RR}=1.06,95 \% \mathrm{CI} 1.04-1.09, p$ not provided $)$ increased risk of NHP and that the highest level of symptoms on both of these scales increased the risks further. Gibbons et al. (2002) found that people with dementia with anxiety symptoms were at an increased risk of NHP ( $R R=1.15,95 \%$ CI 1.03-1.28, $p$ value not provided). Gilley et al. (2004) found that certain BPSD increased risk of NHP; both physical aggression $(\mathrm{RR}=2.30$, 95\% CI 1.41-3.76, $p$ value not provided) and hallucinations $(\mathrm{RR}=1.83,95 \%$ CI $1.27-2.63$, $p$ value not provided) were significant; however, delusions were not $(\mathrm{RR}=0.81,0.56-1.18, p$ not provided). They also found that depression increased the risk of NHP, $(\mathrm{RR}=1.04,95 \% \mathrm{CI}$ 1.01-1.08, $p$ not provided). Scarmeas et al. (2007) found that any "disruptive behavior" increased the risk of NHP $(\mathrm{HR}=1.47,95 \%$ CI $1.10-1.97, p$ not provided). Kunik et al. (2010) found that a person with dementia becoming aggressive significantly increased the risk of NHP $(\mathrm{HR}=2.98,95 \% 1.05-$ $8.49, p=0.004$ ).

Smith et al. (2001) found that "disruptive behaviors" were not significantly associated with NHP (data not provided in paper).

CASE-CONTROL STUDIES

Six studies reported on BPSD as a risk factor for NHP. Four of these (Steele et al., 1990; Lieberman and Kramer, 1991; de Vugt et al., 2005; Coehlo et al., 2007) were entered into a meta-analysis (see 
Figure S10) and people with dementia who were placed in a nursing home had significantly higher BPSD symptoms than those who remained in the community $(\mathrm{SMD}=0.25, \mathrm{SE}=0.1, p=0.01)$.

Not included in the meta-analysis, Young et al. (1998) found that people with dementia admitted to a nursing home had significantly more "behavior problems" than those not admitted $(p<0.001)$. Lieberman and Kramer (1991) also assessed "psychiatric problems" and "problem behaviors" in addition to their standardized measurement of BPSD, and found no significant difference in these symptoms between people with dementia who were placed in a nursing home and those who were not.

\section{Physical health}

\section{COHORT STUDIES}

Four studies found physical health factors that increased the risk of NHP. Harboun et al. (2008) found that a hip fracture within 3 years preceding a dementia diagnosis significantly increased the risk of NHP (HR $=2.7,95 \%$ CI 1.1-6.9, $p<0.05)$. Luppa et al. (2012) found that mobility impairment significantly increased the risk of NHP $(\mathrm{HR}=2.6$, $95 \%$ CI 1.29-5.21, $p=0.007$ ). Smith et al. (2000) found that a change in the number of comorbidities between pre- and post-diagnosis increased the risk of NHP (RR $=1.91,95 \% \mathrm{CI}$ and $p$ value not provided; no details were provided on the nature of these changes). However, Buhr et al. (2006) found that people with dementia with fewer patient comorbidities were at a significantly increased risk of NHP $(\mathrm{RR}=1.16,95 \% \mathrm{CI} 1.12=1.20$, $p$ value not provided).

Five studies identified certain physical health factors that did not influence the risk of NHP. Gilley et al. (2004) found that neither mobility nor urinary incontinence increased or decreased the risk of NHP (mobility: $\mathrm{RR}=1.02,95 \%$ CI $0.85-1.23, p$ value not provided; urinary incontinence: $\mathrm{RR}=1.17,95 \% \quad 0.72-1.90, p$ value not provided). Smith et al. (2001) found that neither comorbidities nor extrapyramidal symptoms increased or decreased the risk of NHP (data not reported by study authors). Smith et al. (2000) found that the number of hospitalizations or doctor visits did not increase or decrease the risk of NHP (data not reported by study authors). Luppa et al. (2012) found that neither visual nor hearing impairment significantly increased the risk of NHP (visual impairment: $\mathrm{HR}=1.45,95 \%$ CI $0.85-2.49, p=0.18$; hearing impairment: $\mathrm{HR}=0.69,95 \%$ CI $0.41-1.17, p=0.17)$. Andel et al. (2007) provided no $p$-values for any of the following conditions, but reported that they were non-significant risk factors: arthritis $(\mathrm{HR}=0.85)$; cancer $(\mathrm{HR}=0.93)$; diabetes $(\mathrm{HR}=1.05)$; chronic obstructive pulmonary disease/emphysema ( $\mathrm{HR}=$ $0.85)$; heart disease $(\mathrm{HR}=0.96)$; incontinence $(\mathrm{HR}=1.14)$; and stroke $(\mathrm{HR}=1.00)$.

\section{CASE-CONTROL STUDIES}

Two studies looked at whether physical health factors were a risk for NHP. Scott et al. (1997) found that, in a univariate analyses, a decline in "locomotion" was associated with NHP ( $p=$ $0.011)$. However, when "locomotion" was entered into the final model of NHP prediction, it was no longer significant (no data reported by the study authors). Lieberman and Kramer (1991) found no significant difference in "medical problems" and "neurological signs" between people with dementia placed in a nursing home and those who were not.

\section{Carer mental health-depression}

\section{COHORT STUDIES}

Five studies found that carer depression did not influence the risk of NHP: four (Yaffe et al., 2002; Gaugler et al., 2003; Gilley et al., 2005; Buhr et al., 2006) used hazard ratios and one (Gaugler et al., 2000) reported risk ratios. In the studies that used hazard ratios, the data were not reported by the study authors. Gaugler et al. (2000) found that carer depression did not increase or decrease the risk of NHP $(\mathrm{RR}=0.97,95 \% \mathrm{CI}$ and $p$ value not provided).

\section{CASE-CONTROL STUDIES}

Two studies investigated whether carer depression was a risk factor for NHP. Whitlatch et al. (1999) found that higher levels of carer depression significantly increased the odds of NHP (OR 1.01, $p<0.05)$. Coehlo et al. (2007) found that carers of people with dementia placed in a nursing home showed significantly more depressive symptoms than those that were not admitted to a nursing home $(p<0.001)$.

\section{Other carer risk factors}

\section{COHORT STUDIES}

Buhr et al. (2006) found that lower life satisfaction amongst carers increased the risk of NHP for people with dementia $(\mathrm{RR}=1.52,95 \% 1.27-1.81$, $p$ value not provided). Gaugler et al. (2003; 2005) reported that poor carer health increased the risk of NHP (HR $=1.44$, no $95 \%$ CI provided, $p=$ $0.004)$ and that carer unmet need at the second highest level increased the risk of NHP (HR = 1.18 , no $95 \%$ CI provided, $p=0.05$ ). Conversely, they found that carer IADL limitations (at any level) did not influence the risk of NHP (IADL 
limitations $=0.1-1, \mathrm{HR}=1.11$, no $95 \% \mathrm{CI}$ provided, $p=0.26$; IADL limitation $=1.1-2$, $\mathrm{HR}=0.9$, no $95 \%$ CI provided, $p=0.38$; IADL limitations $=2.1-8, \mathrm{HR}=1.12$, no $95 \%$ CI provided, $p=0.27$ ) but that, if IADLs had worsened ( 1 activity or more) during a 6-month interval, this significantly decreased the risk of NHP $(\mathrm{HR}=0.68$, no 95\% CI provided, $p=0.01)$.

Harboun et al. (2008) found that help provided by carers did not influence the risk of NHP for people with dementia $(\mathrm{HR}=1,95 \%$ CI 0.6-1.7, $p$ value not provided). Gilley et al. (2005) found that carer positive affect did not influence the risk of NHP (data not reported by study authors) and that caregiving satisfaction significantly decreased the risk of NHP $(\mathrm{HR}=0.929,95 \%$ CI $0.88-$ 0.98, $p<0.01$ ). Spruytte et al. (2001) found that neither the number of caregiving tasks nor carer satisfaction from caregiving influenced the odds of NHP (no data reported by study authors) and that a higher quality of caregiving relationship significantly decreased the odds of NHP (OR = 0.92, no $95 \%$ CI provided, $p=0.02$ ).

\section{CASE-CONTROL STUDIES}

Six studies investigated other carer risk factors for NHP, five of which indicated a number of significant findings. Coehlo et al. (2007) found that carers of people with dementia placed in a nursing home had significantly poorer health $(p<0.05)$ and significantly more negative caregiver experiences $(p<0.05)$ than carers of those who remained at home. De Vugt et al. (2005) found that carers of people with dementia placed in a nursing home were significantly more distressed $(p=0.022)$ and had a significantly lower sense of competence $(p=0.032)$ at baseline. However, there was no significant difference in mood between carers of people with dementia who had been placed and those whose care recipients were not placed ( $p$ value not provided by study authors). Lieberman and Kramer (1991) found that caregiver stress (indicated by the presence of various family problems) was significantly higher in carers of people with dementia placed in nursing homes ( $p<0.001)$. Young et al. (1998) found that carers' personal expectations (their interpretation of their care responsibility) were significantly higher in carers of individuals who were placed in a nursing home $(p<0.05)$. However, there was no significant difference between carers regarding normative expectations (care with respect to a particular kinship relationship). Carers of individuals placed in a nursing home were also found to have significantly lower attachment to the person placed $(p<0.01)$. Pot et al. (2001) found that carers of placed individuals were significantly more stressed than carers of those not placed $(p<0.05)$. However, they found no significant differences in carer psychological distress or carer extraversion between these groups.

Whitlatch et al. (1999) found that caregiver social support did not increase or decrease the odds of NHP $(\mathrm{OR}=1.01,95 \% \mathrm{CI}$ or $p$ value not provided).

\section{Environmental}

\section{COHORT STUDIES}

Gaugler et al. (2003; 2005) found that utilizing low amounts of "chore" services (1-36 hours in preceding 6 months) was significantly associated with an increased risk of NHP (HR $=1.34$, no 95\% CI provided, $p<0.001)$. However, medium (37-103 hours), high (104+ hours), or an increased amount of chore service utilization (an increase of 48 hours over 6 months or more) did not influence the risk of NHP (medium, HR $=1.04$, no $95 \%$ CI provided, $p=0.71$; high, $\mathrm{HR}=1.21$, no $95 \%$ CI provided, $p=0.12$; increase, $\mathrm{HR}=1.17$, no $95 \%$ CI provided, $p=0.21)$. They also found that utilizing adult day care services in the low (1-30 days in preceding 6 months) or high (78+ days) categories significantly increased the risk of NHP (Low: $H R=1.30$, no $95 \%$ CI provided, $p<0.001$; High: $\mathrm{HR}=1.30$, no $95 \%$ CI provided, $p=0.02)$. Neither medium (31-77 days) nor an increased amount of adult day service utilization (increase of 24 days over 6 months or more) was associated with a risk of NHP (medium, HR = 1.07, no $95 \%$ CI provided, $p=0.54$; increased, $\mathrm{HR}=1.02$, no $95 \%$ CI provided, $p=0.91)$. Utilizing medium amounts of personal care (61207 hours in preceding 6 months) significantly decreased the risk of NHP (HR $=0.78$, no $95 \%$ CI provided, $p=0.03$ ). However, low, high, or an increased amount of personal care utilization was not associated with the risk of NHP.

Gaugler et al. (2000) found that none of paid help $(R R=1.02)$, amount of family help $(R R=1)$, family support - sitting with relative $(R R=1.10)$, and family support - taking relative out $(\mathrm{RR}=1.06)$ influenced the risk of NHP (95\% CI or $p$ values not provided). However, they also found that family support with ADLs and with overnight respite significantly decreased the risk of NHP (ADLs: $\mathrm{RR}=0.69$, no $95 \%$ CI values provided, $p<0.01$; overnight respite: $\mathrm{RR}=0.59$, no $95 \% \mathrm{CI}$ values provided, $p<$ 0.01 ). Spruytte et al. (2001) found that lower costs of care did not influence the risk of NHP (no data reported by study author), but that home adaptations significantly decreased the odds of 
NHP (OR $=0.1,95 \%$ CI not provided, $p=0.006)$. Harboun et al. (2008) found that use of health and social care services significantly decreased the risk of NHP (HR $=0.5,95 \%$ CI $0.3-0.9, p<0.05)$.

\section{CASE-CONTROL STUDIES}

Two studies assessed whether environmental factors were risk factors for NHP. Whitlatch et al. (1999) found that utilizing in-home respite assistance and out-of-home 24-hour respite assistance significantly increased the odds of NHP (in-home: $\mathrm{OR}=1.5,95 \%$ CI not provided, $p<0.05$; out-of-home: $\mathrm{OR}=2.22,95 \% \mathrm{CI}$ not provided, $p<0.05)$. However, utilizing day care respite did not impact on the odds of NHP (OR $=1.16$, $95 \% \mathrm{CI}$ or $p$ value not provided). Lieberman and Kramer (1991) found that there was no significant difference in overall service utilization between those who were placed in a nursing home and those who were not $(p=0.16)$. However, when split into component services, three services were found to show significant differences: those people who were placed in a nursing home were utilizing more healthcare services $(p<0.05)$, fewer homemaker services $(p<0.05)$, and fewer medical clinics $(p<$ $0.05)$ than those who remained in the community. Data on the other component services were not reported by the study authors.

\section{Other}

\section{COHORT STUDIES}

Two studies reported other factors that increased the risk of NHP. Smith et al. (2001) found that "unsafe behaviors" increased the risk of NHP $(\mathrm{RR}=2.38,95 \%$ CI $1.65-3.45, p$ value not provided). Spruytte et al. (2001) found that a higher "level of functioning" of a person with dementia significantly increased the odds of NHP (OR = 1.09 , no $95 \%$ CI provided, $p=0.027$ ).

\section{Discussion}

The causes of care home admission for people with dementia are multifactorial and often the result of complex interactions between characteristics of the person with dementia, their caregivers, service providers, and the environment. Many of these risk factors are potentially modifiable and, as such, could be targeted by interventions to reduce the risk of institutionalization. We have collated and analyzed the available evidence with regard to these risk factors and make tentative recommendations as to how these might be addressed by community services, with the aim of supporting people with dementia and their carers to remain living and coping within their own homes for as long as possible. This is the first detailed systematic review and meta-analyses of a large field of research in this area and, as such, provides important information as to the factors most consistently linked with nursing home placement, which might therefore be targeted by interventions, and to those where findings are less consistent and where further research is necessary.

An expected and robust finding, borne out by meta-analyses, is that poorer cognition is consistently linked with an increased risk of nursing home placement for people with dementia. Cognitive enhancement strategies, both pharmacological and non-pharmacological, might therefore be expected to delay institutionalization. There is some evidence that this is so where pharmacological interventions are concerned (Lopez et al., 2009), but no such evidence as yet for cognitive stimulation therapy and the latter may be an important area for future research. Furthermore, the increased risk associated with more severe cognitive impairment suggests a need for greater surveillance as dementia progresses, so that interventions can be targeted effectively toward other unmet needs and risk factors for institutionalization in the most vulnerable.

One might reasonably predict an association between increased functional dependence and risk of institutionalization and, indeed, three of our four meta-analyses did demonstrate a significant association between impairments in activities of daily living or instrumental activities of daily living and risk of nursing home placement. However, a fourth meta-analysis and a number of individual studies reviewed did not find this association. This variability might relate, to some extent, to differences between studies in the specific ADL analyzed, and future research could usefully focus on particular $\mathrm{ADL}$ in an attempt to elucidate the most important functional limitations leading to nursing home placement. Variability in ADL findings might also result from the fact that affected activities tend to vary by stage of dementia, with different implications for quality of life at each stage (Giebel et al., 2015). However, it is also quite conceivable that institutionalization is driven less by the functional dependency itself and more by the extent to which this need can be met and appropriate interventions provided for the person with dementia and his/her caregiver within their own home.

The studies reviewed that measured the use of various support services are of interest in this regard, yet their results must be analyzed with caution if conceptualizing service use as a proxy measure of need. Although a high level of service 
use is likely to reflect a high level of need, the converse is not necessarily true and a low level of service use might reflect either minimal need or an unspecified level of unmet need. This might explain the findings by Gaugler et al. (2003; 2005) that a low level of domestic chore service use and both high and low (but not medium) levels of adult day care service use were associated with an increased risk of institutionalization, whereas a medium (but not high or low) level of personal care service utilization appeared to decrease this risk. Similarly, Lieberman and Kramer (1991) found that individuals placed in nursing homes had been accessing lower levels of 'homemaker' services prior to admission than those who remained in the community, and Whitlatch et al. (1999) found an increased risk of institutionalization amongst those who had been in receipt of inhome respite assistance and out-of-home 24-hour respite services, presumably representing the most dependent individuals.

It is therefore incumbent upon community support services not only to recognize vulnerability amongst those individuals requiring the highest levels of social support, but also to ensure that all levels of need are properly assessed and that appropriate interventions are offered to meet even low-to-medium levels of identified need which, without such support, could result in premature institutionalization. Some studies of case management, aimed at enhancing the coordination between different agencies involved in community dementia care, have shown delays in time to institutionalization, but findings have been inconsistent and there is a need for further research to establish the most effective components of this approach (Reilly et al., 2015).

It is acknowledged that caring for people with dementia is generally associated with high levels of psychological distress and reduced physical health in caregivers (Ballard et al., 2009; Afram et al., 2014). The former study in eight European countries also noted both the influence of caregiver burden and the inability of the informal caregiver to care for the patient as reasons for admission. It is perhaps not surprising, therefore, that our review and meta-analysis show caregiver burden to be significantly associated with an increased risk of care home placement for people with dementia. Some of the more specific caregiver factors that seemed to increase risk of institutionalization were increased role captivity, poorer general health, higher stress, a poorer caregiving relationship, and lower attachment to the person with dementia. Findings with regard to carer depression and satisfaction were more equivocal, with some studies reporting a higher risk of placement in those with lower satisfaction and more depression and others reporting no such association. Further research is therefore required in these areas. Higher levels of carer stress were noted in cases where people with dementia were placed in nursing homes and this can often be the single determining factor for this transition.

It is generally accepted that BPSD are a major cause of nursing home placement for people with dementia, and the findings of our review and meta-analyses confirm the significant role played by this diverse group of symptoms. Among the more specific symptoms found to increase the risk of institutionalization were physical aggression, psychosis, anxiety, hallucinations, and depression. BPSD exemplify the complex patient-caregiverenvironment interactions that so often result in care home admissions and, as such, are a key target for interventions aimed at reducing both the distress experienced by those involved and the risk of institutionalization. A pragmatic approach to the assessment and management of these symptoms should encompass the bio-psycho-social sphere (Kales et al., 2015), and the drive to reduce inappropriate prescribing of antipsychotic medications (Banerjee, 2009), coupled with an increasing appreciation of the overall importance of these symptoms, led to the publication of best practice guidance in the UK (Alzheimer's Society, 2011). Multi-disciplinary interventions by specialist teams at lower levels of disturbance might prevent deterioration and delay nursing home placement (Brodaty et al., 2003).

Several studies have examined the influence of physical health factors on risk of institutionalization in people with dementia, with mixed findings. Hip fracture, reduced mobility, and multiple comorbidities may be risk factors for care home placement, but findings were inconsistent between studies and meta-analysis was not possible owing to the differing measures used. Nevertheless, it seems intuitively appropriate that specialist teams supporting people with dementia and their caregivers should focus not only on psychological and social care needs, but also on physical health and wellbeing, to detect and manage both acute and chronic medical conditions, including pain, and to minimize the risk of polypharmacy in the elderly and cognitively impaired, developing the early work of Jolley and Arie (1992). Such interventions might reduce the risks of BPSD, delirium, and hospital admission (Toot et al., 2013).

Finally, the geographical location of studies may have shaped the findings. Verbeek et al. (2015) found caregiver burden and ADL dependence were common factors across eight European countries. However, they concluded that admission to nursing 
home care is context-specific, given wide variation in factors associated with institutionalization across countries.

\section{Limitations}

Studies included used a wide variety of measures across different domains and it was not always possible to compare these directly. Hence, we were unable to draw firm conclusions about the significance or otherwise of certain potential risk factors for care home placement. Nevertheless, we were able to combine other measures with sufficient confidence to carry out meta-analyses of a number of potential risk factors with apparently robust findings.

Quality of the individual studies varied, but we believe that our use of a rating scale to assign levels of evidence to each study and include only those independently rated by our reviewers as high quality will have served to maximize the reliability of the findings of this review.

We acknowledge that our inferences with regard to interventions that might help to reduce the risk of nursing home placement must be regarded as tentative and that, in many cases, further studies will be required to test these assumptions. Nevertheless, we believe that the identification of potentially modifiable risk factors for institutionalization provides fertile ground for such studies to be designed and conducted.

Additionally, studies in this review were conducted in several countries and regions where a range of state-supported care or services could be available. Some of the inconsistencies in the analyses presented in this paper could be explained by the lack of uniformity of services that carers can receive. Also, in most cases we did not know whether studies took place in any rural areas, therefore the lack of data from rural areas suggests more research studies are required in this area.

\section{Conclusions}

Supporting people with dementia and their carers to live and cope within their own homes for as long as possible is a valid aim for services with the remit of helping people to live well with dementia. This review suggests that poorer cognition, BPSD, and caregiver burden consistently increase the risk of nursing home placement for these individuals. We propose therefore that specialist multidisciplinary teams should focus on cognitive enhancement strategies, assessment and management of BPSD, and carer education and support in order to tackle these potentially modifiable risk factors. Further, ongoing research will be essential to selecting the most effective strategies for delivering these aims, as well as to elucidating the potential significance of other risk factors, such as carer depression and impairments in individual ADL, and the complex relationship between functional dependencies and service provision, where study findings to date have been more diverse.

\section{Conflict of interest}

None.

\section{Description of authors' roles}

S. Toot performed literature search, review of studies, data extraction, statistical calculations, wrote drafts of manuscript, and preparation for submission. T. Swinson performed literature search, review of studies, data extraction, statistical calculations, and wrote drafts of manuscript. M. Devine performed review of studies, data extraction, writing paper, and preparation for submission. D. Challis gave expert guidance on context and content, and edited the manuscript. $M$. Orrell gave the original idea, supervised research assistants and project, and edited the manuscript

\section{Acknowledgments}

The authors wish to thank Dr. Amy Streater, William Pearson and Maria Long for assistance with carrying out the literature search.

\section{References}

Afram, B. et al. (2014). Reasons for institutionalization of people with dementia: informal caregiver reports from 8 European countries. Fournal of American Medical Directors Association, 15, 108-116. doi:10.1016/j.jamda.2013.09.012.

Alzheimer's Society (2011). Optimising Treatment and Care for People with Behavioural and Psychological Symptoms of Dementia: A Best Practice Guide for Health and Social Care Professionals. London: Alzheimer's Society.

Alzheimer's Society (2013). Low Expectations: Attitudes on Choice, Care and Community for People with Dementia in Care Homes. London: Alzheimer's Society.

Andel, R., Hyer, K. and Slack, A. (2007). Risk factors for nursing home placement in older adults with and without dementia. Fournal of Aging and Health, 19, 213-228. doi:10.1177/0898264307299359.

Ballard, C. G. et al. (2009). Management of agitation and aggression associated with Alzheimer disease 67. Nature Reviews Neurology, 5, 245-255. doi:10.1038/nrneurol.2009.39. 
Banerjee, S. (2009). The Use of Antipsychotic Medication for People with Dementia: Time for Action. London: Department of Health.

Blessed, G., Tomlinson, B. E. and Roth, M. (1968). The association between quantitative measures of dementia and of senile change in the cerebral grey matter of elderly subjects. British fournal of Psychiatry 114, 797-811. doi:10.1192/bjp.114.512.797.

Brodaty, H., Draper, B. M. and Low, L.-F. (2003). Behavioural and psychological symptoms of dementia: a seven-tiered model of service delivery. Medical fournal of Australia, 178, 231-234.

Buhr, G. T., Kuchibhatla, M. and Clipp, E. C. (2006). Caregivers' reasons for nursing home placement: clues for improving discussions with families prior to the transition. Gerontologist 46, 52-61. doi:46/1/52.

Coehlo, D. P., Hooker, K. and Bowman, S. (2007). Institutional placement of persons with dementia: what predicts occurrence and timing? Fournal of Family Nursing, 13, 253-277. doi:10.1177/1074840707300947.

Cooper, C., Selwood, A. and Livingston, G. (2008). The prevalence of elder abuse and neglect: a systematic review. Age and Ageing. doi:10.1093/ageing/afm194.

Cummings, J. L., Mega, M., Gray, K., Rosenberg-Thompson, S., Carusi, D. A. and Gornbein, J. (1994). The neuropsychiatric inventory: comprehensive assessment of psychopathology in dementia. Neurology, 44, 2308-2314. doi:10.1212/WNL.44.12.2308.

de Vugt, M. E., Stevens, F., Aalten, P., Lousberg, R., Jaspers, N. and Verhey, F. R. J. (2005). A prospective study of the effects of behavioral symptoms on the institutionalization of patients with dementia. International Psychogeriatrics, 17, 577-589. doi:10.1017/S1041610205002292.

Folstein, M. F., Folstein, S. E. and McHugh, P. R. (1975). "Mini-mental state". A practical method for grading the cognitive state of patients for the clinician. Fournal of Psychiatric Research, 12, 189-198. doi:10.1016/0022-3956(75)90026-6.

Gaugler, J. E. et al. (2000). Predictors of institutionalization of cognitively impaired elders: family help and the timing of placement. Fournals of Gerontology. Series B: Psychological Sciences and Social Sciences, 55, 247-255. doi:http://dx.doi.org/10.1093/geronb/55.4.P247.

Gaugler, J. E., Kane, R. L., Kane, R. A., Clay, T. and Newcomer, R. (2003). Caregiving and institutionalization of cognitively impaired older people: utilizing dynamic predictors of change. Gerontologist, 43, 219-229. doi:10.1093/geront/43.2.219.

Gaugler, J. E., Kane, R. L., Kane, R. A. and Newcomer, R. (2005). Early community-based service utilization and its effects on institutionalization in dementia caregiving. The Gerontologist, 45, 177-185. doi:10.1093/geront/45.2.177.

Gibbons, L. E. et al. (2002). Anxiety symptoms as predictors of nursing home placement in patients with Alzheimer's disease. Fournal of Clinical Geropsychology, 8, 335-342.

Giebel, C. M., Sutcliffe, C. and Challis, D. (2015). Activities of daily living and quality of life across different stages of dementia: a UK study. Aging $\mathcal{E}$ Mental Health, 19, 63-71. doi:10.1080/13607863.2014.915920.
Gilley, D. W., Bienias, J. L., Wilson, R. S., Bennett, D. A., Beck, T. L. and Evans, D. A. (2004). Influence of behavioral symptoms on rates of institutionalization for persons with Alzheimer's disease. Psychological Medicine, 34, 1129-1135. doi:10.1017/S0033291703001831.

Gilley, D. W., McCann, J. J., Bienias, J. L. and Evans, D., (2005). Caregiver psychological adjustment and institutionalization of persons with Alzheimer's disease. Fournal of Aging and Health 17, 172-189. doi:10.1177/0898264304274252.

Guerchet, M., Prina, M. and Prince, M. (2013). Policy Brief for Heads of Government: The Global Impact of Dementia 2013-2050 (pp. 1-8). London: Alzheimer's Disease International.

Harboun, M., Dorenlot, P., Cohen, N., Steinhagen-Thiessen, E. and Ankri, J. (2008). Impact of hip fracture, heart failure and weight loss on the risk of institutionalization of community-dwelling patients with dementia. International fournal of Geriatric Psychiatry, 23, 1245-1252. doi:10.1002/gps.2058.

Haupt, M. and Kurz, A. (1993). Predictors of nursing home placement in patients with Alzheimer's disease. International fournal of Geriatric Psychiatry, 8, 741-746. doi:10.1002/gps.930080906.

Jolley, D. and Arie, T. (1992). Developments in psychogeriatric services. In T. Arie (ed.), Recent Advances in Psychogeriatrics, vol. 2. London: Churchill Livingstone.

Kales, H. C., Gitlin, L. N. and Lyketsos, C. G. (2015). Assessment and management of behavioral and psychological symptoms of dementia. BMF, 350, 369-369. doi:10.1136/bmj.h369.

Katz, S., Ford, A. B., Moskowitz, R. W., Jackson, B. and Jaffe, M. W. (1963). Studies of illness in the aged. The index of ADL: a standardized measure of biological and psychosocial function. Fournal of American Medical Association, 185, 914-919. doi:10.1001/jama.1963.03060120024016.

Kunik, M. E. et al. (2010). Consequences of aggressive behavior in patients with dementia. Fournal of Neuropsychiatry and Clinical Neurosciences, 22, 40-47. doi:10.1176/appi.neuropsych.22.1.40.

Lawton, M. P. and Brody, E. M. (1969). Assessment of older people: self-maintaining and instrumental activities of daily living. Gerontologist, 9, 179-186. doi:10.1093/geront/9.3_Part_1.179.

Lawton, M. P., Moss, M., Kleban, M. H., Glicksman, A. and Rovine, M. (1991). A two-factor model of caregiving appraisal and psychological well-being. Fournal of Gerontology, 46, 181-189. doi:10.1093/geronj/46.4.P181.

Lieberman, M. A. and Kramer, J. H. (1991). Factors affecting decisions to institutionalize demented elderly. Gerontologist, 31, 371-374.

Lopez, O. L. et al. (2009). Long-term effects of the concomitant use of memantine with cholinesterase inhibition in Alzheimer disease. Fournal of Neurology, Neurosurgery \& Psychiatry, 80, 600-607. doi:10.1136/jnnp.2008.158964.

Luppa, M. et al. (2012). Predictors of institutionalisation in incident dementia-results of the German study on ageing cognitive and dementia in primary care patients (AgeCoDe 
study). Dementia and Geriatric Cognitive Disorders, 33, 282-288.

Mattis, S. (1988). Dementia Rating Scale (DRS). Odessa, FL: Psychological Assessment Resources.

Morris, J. N. et al. (1997). Comprehensive clinical assessment in community setting: applicability of the MDS-HC. Fournal of American Geriatric Society, 45, 1017-1024. doi:10.1111/j.1532-5415.1997.tb02975.x.

Pot, A. M., Deeg, D. J. H. and Knipscheer, C. P. M. (2001). Institutionalization of demented elderly: the role of caregiver characteristics. International fournal of Geriatric Psychiatry, 16, 273-280. doi:10.1002/gps.331.

Prince, M., Prina, M. and Guerchet, M. (2015). World Alzheimer Report 2013: Fourney of Caring-Analysis of Long-Term Care for Dementia, London: Alzheimer's Disease International.

Reilly, S. et al. (2015). Case management approaches to home support for people with dementia. Cochrane database Systematic Reviews 1, CD008345. doi:10.1002/14651858.CD008345.pub2.

Roth, M., Tym, E. and Mountjoy, C. Q. (1986). CAMDEX. A standardised instrument for the diagnosis of mental disorder in the elderly with special reference to the early detection of dementia. British fournal of Psychiatry, 149, 698-709. doi:10.1192/bjp.149.6.698.

Scarmeas, N. et al., (2007). Disruptive behavior as a predictor in Alzheimer disease. Archives of Neurology, 64, 1755-1761. doi:10.1001/archneur.64.12.1755.

Scott, W. K., Edwards, K. B., Davis, D. R., Cornman, C. B. and Macera, C. A. (1997). Risk of institutionalization among community long-term care clients with dementia. Gerontologist, 37, 46-51. doi:10.1093/geront/37.1.46.

Severson, M. A. et al. (1994). Patterns and predictors of institutionalization in community-based dementia patients. Fournal of American Geriatrics Society, 42, 181185.

Smith, G. E., Kokmen, E. and O'Brien, P. C. (2000). Risk factors for nursing home placement in a population-based dementia cohort. Fournal of American Geriatric Society, 48, 519-525.

Smith, G. E., O'Brien, P. C., Ivnik, R. J., Kokmen, E. and Tangalos, E. G. (2001). Prospective analysis of risk factors for nursing home placement of dementia patients. Neurology, 57, 1467-1473. doi:10.1212/WNL.57.8.1467.

Spruytte, N., Van Audenhove, C. and Lammertyn, F. (2001). Predictors of institutionalization of cognitively-impaired elderly cared for by their relatives.
International fournal of Geriatric Psychiatry, 16, 1119-1128. doi:10.1002/gps.484.

Steele, C., Rovner, B., Chase, G. A. and Folstein, M. (1990). Psychiatric symptoms and nursing home placement of patients with Alzheimer's disease. The American of Fournal of Psychiatry ,147, 1049-1051. doi:10.1176/ajp.147.8.1049.

Sury, L., Burns, K. and Brodaty, H. (2013). Moving in: adjustment of people living with dementia going into a nursing home and their families. International Psychogeriatrics, 25, 867-876. doi:10.1017/S1041610213000057.

Toot, S., Devine, M., Akporobaro, A. and Orrell, M. (2013). Causes of hospital admission for people with dementia: a systematic review and meta-analysis. Fournal of American Medical Directors Association, 14, 463-470, doi: 10.1016/j.jamda.2013.01.011.

Verbeek, H. et al. (2015). Inter-country exploration of factors associated with admission to long-term institutional dementia care: evidence from the RightTimePlaceCare study. Fournal of Advanced Nursing, 71, 1338-1350. doi:10.1111/jan.12663.

Weintraub, S. (1986). The record of independent living: an informant-completed measure of activities of daily living and behavior in elderly patients with cognitive impairment. American fournal of Alzheimers Disease \& Other Dementias, 1, 35-39. doi:10.1177/153331758600100210.

Wells, G. et al. (2012). The Nervcastle-Ottawa Scale (NOS) for Assessing the Quality of Nonrandomized Studies in Meta-Analyses. Available at: http//www.ohri.ca/programs/ clinical_epidemiology/oxford.asp.

Whitlatch, C. J., Feinberg, L. F. and Stevens, E. J. (1999). Predictors of institutionalization for persons with Alzheimer's disease and the impact on family caregivers. Fournal of Mental Health $\mathcal{E}$ Aging, 5, 275-288.

WHO (2012). Dementia: A Public Health Priority. Geneva, Switzerland: WHO publication.

Wilkinson, I.M. and Graham-White, J. (1980). Psychogeriatric dependency rating scales (PGDRS). A method of assessment for use by nurses. British fournal of Psychiatry, 137, 558-565. doi:10.1192/bjp.137.6.558.

Yaffe, K. et al. (2002). Patient and caregiver characteristics and nursing home placement in patients with dementia. fAMA, 287, 2090-2097. doi:10.1001/jama.287.16.2090.

Young, R. F., Kosloski, K. and Montgomery, R. J. (1998). Psychosocial factors in institutionalization of Alzheimer's patients. Fournal of Clinical Geropsychology, 4, 241-251. 